\title{
Why and How Much Brouwer's Fixed Point Theorem Fails in Noncompact Setting?
}

\author{
Emanuele Casini and Kazimierz Goebel
}

\begin{abstract}
The celebrated Brouwer's Fixed Point Theorem is dated in 1912. Its extension to compact set setting in Banach spaces due to Schauder appeared in 1930. Immediately it raised the question whether the Theorem can be extended to noncompact setting. The works of Kakutani, Klee, Benyamini and Sterfeld, Sternfeld and Lim solved the qualitative part of the problem. Lack of compactness makes the statement of the theorem false. However, there are some quantitative aspects of the question. The two basic are called minimal displacement problem, and optimal retraction problem. The aim of this article is to present the historical back ground and possibly, up to date state of investigations in this field. A list of open problems with comments will be discussed.
\end{abstract}

Mathematics Subject Classification (2010). Primary 47H10.

Keywords. Brouwer's Fixed Point Theorem, fixed point free mappings, minimal displacement problem, optimal retraction problem.

\section{Elements of History}

The history of the Brouwer's Fixed Point Theorem has almost one hundred years. The officially most cited paper is [9] from 1912. However historians of mathematics and interested specialists found that the main ideas behind this theorem has been known to some time before Brouwer announced his result. It is believed that the fact was known to Poincaré. The result equivalent to Brouwer's Theorem but formulated in a different form can be found in the paper by Bohl [6]. It is not our aim to discuss the beginnings of the theory. The aim of this article is to present a direction of research which has its roots in Brouwer's Theorem but appeared later with the development of functional analysis and investigations of spaces of infinite dimension.

In the most commonly used present mathematical language the classical theorem reads: 
Theorem 1 (Brouwer's Fixed Point Theorem). The unit ball $B^{n} \subset \mathbb{R}^{n}$ has topological fixed point property.

It means that for any continuous mapping $T: B^{n} \rightarrow B^{n}$ there exists a point $x \in B^{n}$ such that $x=T x$, a fixed point of $T$. This property, (generally abbreviated as fpp) is topologically invariant. All sets homeomorphic to $B^{n}$ share the fixed point property. Since any closed convex and bounded subset $C$ of a finite dimensional Banach space $X$ is homeomorphic to a ball of certain dimension all such sets have fpp. Also this property is inherited by all the retracts of $C$. Let us recall that a subset $D \subset C$ is a retract of $C$ if there exists a continuous mapping (a retraction) $r: C \rightarrow D$ such that $x=r x$ for all $x \in D$. In other words if the identity mapping on $D$ can be continuously extended to a mapping $r: C \rightarrow D$.

The original Brouwer's proof was based on the degree theory, the method which was still not so well developed at that time. The very clear and rigorous proof has been given by Knaster, Kuratowski and Mazurkiewicz [23]. The method was based on combinatorial Sperner's Lemma and on a topological fact known today as KKMLemma. Later other proofs appeared. The most recent and the most elementary ones, based on relatively simple analytical methods, can be found in [27], [18], [24], .

Soon after Brouwer's Theorem has been announced the new questions appeared. It was the time of the vigorous development of methods of functional analysis, so the natural problem raised was about the possibility of the extension of this result to the case of infinitely dimensional spaces.

The most famous result is:

Theorem 2 (Schauder's Fixed Point Theorem). Any convex and compact subset $C$ of a Banach space $X$ has topological fixed point property.

The obvious difference is that in infinite dimensional Banach spaces, bounded and closed sets do not need to be compact. Especially it is the case of balls. The proof of Schauder's Theorem is based on the fact that any continuous self-mapping of $C$ can be uniformly approximated by finite dimensional mappings for which Brouwer's Theorem works.

The aim of this article is to discuss the next step. What happens in the noncompact setting? It occurs that both results become false. But, for many years a lot of nontrivial questions has been raised and some of them are still open. In what extend the lack of compactness influence existence of continuous fixed point free mappings? How "regular" such mappings can be? How far they can move all the points? How much the numerous equivalents of Brouwer's Theorems fail in infinite dimensional Spaces? Can we measure "the rate of failure?

We shall try to introduce the reader to the present state of the subject keeping more or less historical order. We often resign from the rigorous proofs giving the reader only directions and hints for the methods used. Also we often resign from presenting in details the best results and estimates. Instead we show examples which seem to be simple but represent well the flavour of the field. Chapters devoted to the subject containing more detailed discussion can be found in the following books: [13], [19], [10]. 


\section{Preliminaries}

Let $X$ be a Banach space of finite or infinite dimension. Let $B$ stays for the unit ball and $S$ for the unit sphere. If the dimension $X$ is finite $\operatorname{dim} X=n$ we use $B=B^{n}$ and $S=S^{n-1}$.

The Brouwer's Theorem has a number of equivalent statements. We shall need two of them. A larger collection of equivalents is to be found in [12]

Theorem 3 (No retraction theorem). $S^{n-1}$ is not the retract of $B^{n}$.

Equivalence of this two facts is proved by the following observations. First, suppose that $R: B \rightarrow S$ would be a retraction of the ball $B^{n}$ onto the sphere $S^{n-1}$. Then the mapping $T=-R: B \rightarrow B$ must be fixed point free On the other hand, suppose that $T: B \rightarrow B$ is such that for all $x \in B^{n}, x \neq T x$. Then we can extend this mapping to the doubled unit ball $2 B^{n}$ by putting

$$
T x=(2-\|x\|) T\left(\frac{x}{\|x\|}\right) \text { for } x \in 2 B \backslash B .
$$

Now, the modified mapping $T: 2 B^{n} \rightarrow B^{n}$ is again fixed point free and transforms the doubled sphere $2 S^{n-1}$ into the origin. Modifying the mapping again by setting $\widetilde{T} x=\frac{1}{2} T(2 x)$ we get the fixed point free mapping $\widetilde{T}: B^{n} \rightarrow B^{n}$ satisfying $\widetilde{T}(S)=$ $\{0\}$ and consequently the retraction $R: B^{n} \rightarrow S^{n-1}$,

$$
R x=\frac{x-\widetilde{T} x}{\|x-\widetilde{T} x\|} .
$$

The second equivalent formulation is:

Theorem 4 (Non contractibility theorem). The sphere $S^{n-1}$ is not contractible to a point.

The above means that identity mapping on $S^{n-1}$ is not homotopic to a constant map. More precisely, there is no homotopy $H:[0,1] \times S^{n-1} \rightarrow S^{n-1}$ such that $H(0, x)=x$ for all $x \in S^{n-1}$ and $H(1, x)=z$ where $z$ is a given point in $S^{n-1}$.

The equivalence of noncontractibility and the lack of retractions comes from the following. Suppose there is a retraction $R: B^{n} \rightarrow S^{n-1}$ then the formula

$$
H(t, x)=R((1-t) x)
$$

defines the homotopy joining the identity map with the constant map $H(1, x)=z=$ $R(0)$. On the other hand, supposing that $H:[0,1] \times S^{n-1} \rightarrow S^{n-1}$ is a homotopy joining the identity with a constant map $H(1, x)=z$, we can construct a retraction $R: B^{n} \rightarrow S^{n-1}$. This, for any $0 \leq r<1$ can be defined by the formula

$$
R x=\left\{\begin{array}{cc}
z & \text { if }\|x\| \leq r \\
H\left(\frac{1-\|x\|}{1-r}, \frac{x}{\|x\|}\right) & \text { if } r<\|x\| \leq 1
\end{array} .\right.
$$

To end the preliminaries, let us observe that all the constructions presented above do not use the fact that we are working in the finite dimensional space $\mathbb{R}^{n}$. Actually we can formulate a statement called by some specialists "trivial theorem". 
Theorem 5 (trivial theorem). For any Banach space $X$ the following three statements are equivalent:
A) The unit ball $B$ has the fixed point property,
B) The unit sphere $S$ is not the retract of $B$,
C) $S$ is not contractible.

The equivalence itself, as it was shown, is a simple matter. The classical result of Brouwer implies that all three statements are true for $X=\mathbb{R}^{n}$ and consequently for all the Banach spaces of finite dimension. The rest of the article is devoted to the discussion of the fact that all three statements fail to be true for if $\operatorname{dim} X=\infty$. We shall discuss and justify variants and modifications of the following

Theorem 6. For any Banach space $X$ of infinite dimension the following three statements are true and equivalent:

A) There exists a continuous mapping $T: B \rightarrow B$ without fixed points

B) The unit sphere $S$ is the retract of $B$,

C) $S$ is contractible.

Equivalence has been already shown. The validity of three statements was proved step by step within several years by several authors.

\section{Ulam's question and Kakutani's solution}

The first questions about the failure of Brouwer's Theorem were probably raised just after Schauder's Theorem has been published in 1930. There are not many informations about examples, constructions and ideas of mathematicians of that time. Probably the first written sign that mathematicians at that time were interested in the problem is the question raised by S. Ulam around 1935. In the famous collection of mathematical problems known as "The Scottish Book" (for references see [26]) Ulam asked: "Can one transform continuously the solid sphere of a Hilbert space into its boundary such that the transformation should be the identity on the boundary of the ball". There is also a note saying: "There exists a transformation with the required property given by Tychonoff". Unfortunately the mentioned example is not described and probably unpublished, it was only known to a narrow circle of people.

The first widely known examples solving this problem come from the paper of S. Kakutani published in 1943 [17]. There are two steps in Kakutani's construction. First step is to prove that there are continuous mappings $T: B \rightarrow B$ having no fixed points.

Example 1. Consider the standard model of a Hilbert space, the space $l^{2}$. Define the mapping $T: B \rightarrow S \subset B$ by putting for any $x=\left(x_{1}, x_{2}, x_{3}, \ldots\right) \in l^{2}$,

$$
T x=\left(\sqrt{1-\|\mathbf{x}\|^{2}}, x_{1}, x_{2}, x_{3}, \ldots\right) \text {. }
$$


The mapping is fixed point free, since $T x=x$ implies $x=0=(0,0,0, \ldots)$ but $T 0=\mathbf{e}_{1}=(1,0,0, \ldots)$.

The above mapping is obviously continuous and even more, it's continuity is uniform. The modulus of continuity can be easily evaluated from the easily justified inequality,

$$
\|T x-T y\| \leq \sqrt{2\|x-y\|}+\|x-y\| .
$$

The structure of the mapping $T$ is simple. It is an isometry (shift) perturbed by a one dimensional mapping. It is also a technicality to check that $T$ has the minimal displacement zero, or in other words, an approximate fixed point which means that

$$
\inf [\|x-T x\|: x \in B]=0 .
$$

There are some modifications of this example toward finding fixed point free mappings being "more regular" For example, defining for any $0<\varepsilon \leq 1$ the mapping

$$
T_{\varepsilon} x=\left(\epsilon(1-\|x\|), x_{1}, x_{2}, x_{3}, \ldots\right),
$$

we get the same conclusion but the mapping is lipschitzian,

$$
\|T x-T y\| \leq \sqrt{1+\varepsilon^{2}}\|x-y\| .
$$

Once we have a fixed point free mapping $T: B \rightarrow B$ we can construct a retraction $R: B \rightarrow S$ applying the following recipe. For any $x \in B$, find $T x$ and follow the straight half line beginning at $T x$ and passing through $x$ until you reach a point on the the sphere $S$. Define the value of the retraction $R x$ as this point. More precisely put,

and

$$
u(x)=\frac{x-T x}{\|x-T x\|}
$$

$$
R x=x+\lambda(x) u(x),
$$

where the coefficient $\lambda(x) \geq 0$ is selected to satisfy $\|R x\|=1$. Simple calculations show that,

$$
\lambda(x)=-(x, u(x))+\sqrt{1-\|x\|^{2}+(x, u(x))^{2}} .
$$

The natural question arises. Can one repeat the same argument and prove that in any Banach space the unit sphere $S$ is the retract of the unit ball $B$. The basic question is, can one define a continuous mapping $T: B \rightarrow B$ with no fixed points? Once we have it we still can not use directly the scheme proposed by Kakutani. The unit sphere in a Banach space can contain convex subsets of positive diameter. It may happen that for a point $x \in S$ the whole segment joining $x$ and $T x$ is contained in $S$. In such a case the recipe from Kakutani's example described by (3.1) does not work directly. However, after a simple modification it works. First we can use the formula (2.1) to define the fixed point free mapping $\widetilde{T}: B \rightarrow B$ such that $\widetilde{T}(S)=\{0\}$. Then we can follow Kakutani's construction of $R: B \rightarrow S$. Since, in the case of arbitrary Banach space, we do not get exact formula for $\lambda(x)$, the only technicality is to prove its continuity. 
Also, once we have a fixed point free mapping $\widetilde{T}: B \rightarrow B$ which sends $S$ into the origin, we can define the retraction of the ball onto sphere in a simpler way. It is enough to put

$$
\widetilde{R} x=\frac{x-\widetilde{T} x}{\|x-\widetilde{T} x\|} .
$$

Thus the only task to define a retraction of $B$ onto $S$ is to find a fixed point free self-mapping on the ball. In what follows we will show some concrete examples. Now let us pass to the general result.

\section{Klee's results}

The full solution to the Ulam's question came in years 1953 and 1955 after two papers by V. Klee [20] and.[21] Two basic facts can be selected from a number even more general contained in these papers.

Theorem 7. For any infinitely dimensional Banach space $X$ the unit ball $B$ and punctured unit ball $B \backslash\{0\}$ are homeomorphic and there exists a homeomorphism $H: B \rightarrow B \backslash\{0\}$ such that $H$ is the identity on the unit sphere $S$, for all $x \in S$, $x=H x$.

Theorem 8. For any infinitely dimensional Banach space $X$ and for any convex, closed, bounded but noncompact subset $C \subset X$ there exists a fixed point free, continuous mapping $T: C \rightarrow C$.

The first result gives an immediate answer to the retraction problem. Having a mapping $H: B \rightarrow B \backslash\{0\}$ satisfying the above condition we can easily construct the retraction $R: B \rightarrow S$ by putting

$$
R x=\frac{H x}{\|H x\|} .
$$

The only disadvantage of this result is that we do not have any control on regularity of $H$ and consequently $R$. These mappings are not uniformly continuous.

The proof of the second theorem is based on the fact that noncompact convex set $C$ must contain a homeomorphic image of the unbounded interval $[0, \infty)$ imbedded in $C$ as a closed subset. More precisely, there exists an invertible continuous function $\gamma:[0, \infty) \rightarrow C$ having continuous inverse $\gamma^{-1}$. Then, $\Gamma=\gamma([0, \infty))$ is a closed subset of $C$. The function $\gamma^{-1}: \Gamma \rightarrow[0, \infty)$ can be, by virtue of Titze's Theorem extended to the continuous function $\widetilde{\gamma^{-1}}: C \rightarrow[0, \infty)$ such that $\widetilde{\gamma^{-1}}(x)=\gamma^{-1}(x)$ for all $x \in \Gamma$. Now, two observations can be made.

First that $\Gamma$ is the retract of $C$. Indeed the retraction $r: C \rightarrow \Gamma$ can be defined as

$$
r(x)=\gamma\left(\widetilde{\gamma^{-1}}(x)\right) .
$$


Second, that the fixed point free mapping $S: \Gamma \rightarrow \Gamma$ shifting each point $x \in \Gamma$, $x=\gamma(t)$ to the point $S x=\gamma(t+1)$ can be extended as the fixed point free mapping to the whole set $C$ by the formula

$$
\widetilde{S}(x)=\gamma\left(\widetilde{\gamma^{-1}}(x)+1\right) .
$$

Observe that the final mapping $\widetilde{S}$ maps the bounded set $C$ onto the curve $\gamma([1, \infty)) \subset \Gamma$ of infinite lengths. Thus $\widetilde{S}$ can not be uniformly continuous.

Tricks with curves of infinite lengths embedded as a closed set in a bounded convex subset of Banach space can show also other singular behaviors of mappings. Here are sample examples, based on construction from([16]), which illustrate some possibilities.

Example 2. Let $X$ be a nonreflexive Banach space. It is known that nonreflexivity is equivalent to existence of a linear functional $f \in X^{*}$ which does not attain its norm on the unit ball. If we assume that $\|f\|_{X^{*}}=1$ then we have

$$
-1=\inf [f(x): x \in B]<f(x)<\sup [f(x): x \in B]=1 .
$$

Define the function $\Phi: X \rightarrow(0, \infty)$ by setting

$$
\Phi(x)=1+f(x)+2 \max [0,\|x\|-1] .
$$

It is easy to check that $\Phi$ is positive, continuous, convex, with $\inf [\Phi(x): x \in X]=$ $\inf [\Phi(x): x \in S]=0$ and $\lim _{\|x\| \rightarrow \infty} \Phi(x)=\infty$. Moreover, when restricted to the unit ball $B, \Phi$ is affine, $\Phi(x)=1+f(x)$ for $x \in B$. Also $\Phi$ does not take value 0 at any point of $X$ and $\Phi(B)=\Phi(S)=(0,2)$. Now, select a sequence $\left\{x_{n}\right\}, n=$ $0,1,2, \ldots, x_{n} \in S$ such that $\Phi\left(x_{n}\right)=2^{-n}$. Such sequences exist and do not have cluster points. Otherwise $\Phi$ would take value 0 . Consider now the piecewise linear curve $\gamma:(0,1) \rightarrow X$ defined by

$$
\gamma(t)=\left\{\begin{array}{cc}
(1-s) x_{n}+s x_{n+1} & \text { for } t=(1-s) 2^{-n}+s 2^{-(n+1)}, \\
\frac{t+1}{2} x_{0} & \text { for } t \geq 1 .
\end{array}\right.
$$

and put $\Gamma=\gamma((0, \infty))$. Observe that the above parametrization is invertible and that $\Phi(\gamma(t))=t$. For any $r>0$, let $C_{r}$ denotes the sub-level set $C_{r}=[x: \Phi(x) \leq r]$ and $L_{r}$ the strict level set $L_{r}=[x: \Phi(x)=r]$. Thus, all sets $C_{r}$ are bounded closed and convex with $\cap_{r>0} C_{r}=\varnothing$. For any $s \in(0,1)$ define the mapping $T_{s}: C_{r} \rightarrow$ $\Gamma \cap C_{s r} \subset C_{r}$,

$$
T_{s} x=\gamma(s \Phi(x)) .
$$

All mappings $T_{s}, s \in(0,1)$ are continuous. Also, since $\Phi\left(T_{s}(x)\right)=s \Phi(x)<\Phi(x)$. all mappings $T_{s}$ are fixed point free. Moreover, one can observe that the family $T_{s}$ forms a semigroup

$$
T_{s t}=T_{s} \circ T_{t} .
$$

Example 3. With all the notations of the example above proceed with the following recipe. Take arbitrary $a>0$ and for any $x \in X$ consider the half line $l(t), t \geq 0$ defined as

$$
l_{x}(t)=x+t\left(\gamma\left(\frac{1}{2} \Phi(x)\right)-x\right)=x+t\left(T_{\frac{1}{2}} x-x\right) .
$$


Since $\Phi$ is convex and $\lim _{\|x\| \rightarrow \infty} \Phi(x)=\infty$, there exists unique $\bar{t}=\bar{t}(x)>0$ such that $\Phi\left(l_{x}(\bar{t})\right)=\Phi\left(l_{x}(0)\right)+a=\Phi(x)+a$. Define the mapping $H_{a}: X \rightarrow X \backslash C_{a}$ by putting

$$
H_{a} x=l_{x}(\bar{t}(x)) .
$$

Observe that the procedure defining $H_{a}$ is invertible and $H_{a}^{-1}\left(X \backslash C_{a}\right)=X$. Proving the continuity of $H_{a}$ and $H_{a}^{-1}$ is just a technicality. This proves that the whole space $X$ is homeomorphic with the punctured space $X \backslash C_{a}$. Since $C_{2}$ contains the unit ball $B$, slight modification of the construction leads tho the conclusion that $X$ is homeomorphic to $X \backslash B$. Further one can observe that $X \backslash B$, so also $X$, is homeomorphic to the space punctured by one point $X \backslash\{0\}$. We leave the proofs of last statements to the reader.

Example 4. Follow the construction from the last example with $a=0$. This way, we get the continuous map $T=H_{0}: X \rightarrow X$ satisfying $T^{2} x=x$, for all $x \in X$. Thus $T$ is an involution on $X, T^{2}=I$ or in other words $T=T^{-1}$ on $X$. The mapping $T$ is fixed point free. Indeed, the segment joining $x$ and $T x$ contains the point $y=\gamma\left(\frac{1}{2} \Phi(x)\right)$ which differ from both in view of $\Phi(y)=\frac{1}{2} \Phi(x)=\frac{1}{2} \Phi(T x)$.

The constructions of the above type have one disadvantage. Since the bounded set is mapped onto a curve of infinite length the mappings under concern can not be uniformly continuous. We do not have any control on regularity of constructed mappings.

\section{Statement of quantitative questions}

It is believed that the first quantitative questions has been raised in 1973 in ([11]). It was observed that in the all known examples of fixed point free mappings either we have

$$
\inf [\|x-T x\|: x \in C]=0
$$

or we do not have any control of this quantity. The first examples shown in ([11]) have led to quantitative questions. The first is called the minimal displacement problem and the second the optimal retraction problem. The third, the optimal homotopy problem is not so often mentioned but has strong connection with first two.

\subsection{Minimal displacement problem}

For any mapping $T: C \rightarrow C$ the minimal displacement of $T$ is defined as

$$
d(T)=\inf [\|x-T x\|: x \in C] .
$$

Instead of looking for fixed points of $T$ we can restrict ourselves to the problem of finding or evaluating $d(T)$.If $\mathcal{F}$ is a family of mappings we can try to find uniform evaluation

$$
d(\mathcal{F})=\sup [d(T): T \in \mathcal{F}] .
$$


The basic and the most exploited is the case of the class $\mathcal{L}$ of all lipschitzian mappings. Let $\mathcal{L}(k) \subset \mathcal{L}, k \geq 0$ denotes the class of all mappings satisfying the Lipschitz condition with a given constant $k$

$$
\|T x-T y\| \leq k\|x-y\| .
$$

If $k<1, T$ has a unique fixed point and obviously $d(T)=0$. Thus, the case of our interest is $k \geq 1$. Let $T \in \mathcal{L}(k), z \in C$ and $\varepsilon>0$ be given. Consider the equation

$$
x=\left(1-\frac{1}{k+\varepsilon}\right) z+\frac{1}{k+\varepsilon} T x .
$$

The transformation defined by the right hand side of the above is the contraction of class $\mathcal{L}\left(\frac{k}{k+\varepsilon}\right)$. Thus there is unique $x$ satisfying the equation. Then we have

$$
\|x-T x\|=\left(1-\frac{1}{k+\varepsilon}\right)\|z-T x\| \leq\left(1-\frac{1}{k+\varepsilon}\right) \sup [\|z-y\|: y \in C] .
$$

Abstracting of the selection of $z \in C$ and $\varepsilon>0$ we get

$$
d(T) \leq\left(1-\frac{1}{k}\right) r(C)
$$

where

$$
r(C)=\inf _{z \in C} \sup [\|z-y\|: y \in C]
$$

is the Chebyshev radius of $C$. Following the introduced notation we can write

$$
T \in \mathcal{L}(k) \Longrightarrow d(T) \leq\left(1-\frac{1}{k}\right) r(C) \text { or more } d(\mathcal{L}(k)) \leq\left(1-\frac{1}{k}\right) r(C) \text {. }
$$

Observe now, that if the set $D$ is a shifted multiple of $C, D=u+a C$, then $r(D)=$ $\operatorname{ar}(C)$ and for any $T: C \rightarrow C$ the mapping $F: D \rightarrow D$ defined by

$$
F x=a T\left(\frac{x-u}{a}\right)+u
$$

is also of the class $\mathcal{L}(k)$ with $d(F)=a d(T)$. Thus without loss of generality, from now on, we can always assume that we are dealing with sets $C$ of Chebyshev radius one, $r(C)=1$.

The minimal displacement problem for the whole family $\mathcal{L}$ is finally formalized as follows. For any set $C$ we can define the characteristic of minimal displacement. It is the function $\varphi_{C}:[1, \infty) \rightarrow[0, r(C)]$ defined by

$$
\varphi_{C}(k)=\sup [d(k): T: C \rightarrow C, T \in \mathcal{L}(k)] .
$$

Consequently, for the whole space $X$ define the characteristic of the whole space as

$$
\varphi_{X}(k)=\sup \left[\varphi_{C}(k): C \subset X, r(C)=1\right]=\sup \left[\frac{\varphi_{C}(k)}{r(C)}: C \subset X\right] .
$$

To point out the special role of the case of the unit ball $B \subset X$ we usually use the special notation

$$
\psi_{X}(k)=\varphi_{B}(k)
$$


If it is clear from the context we skip the lower indices indicating the set or the space:

Let us list here some basic properties, common for all characteristics $\varphi$ and $\psi$ :

- the ratio $\frac{\varphi(k)}{k-1}$ decreases with $k$,

- the ratio $\frac{k \varphi(k)}{k-1}$ increases with $k$,

- the derivative $\varphi^{\prime}(1)$ exists and $0<\varphi^{\prime}(1) \leq 1$,

- $\varphi^{\prime}(1)\left(1-\frac{1}{k}\right) \leq \varphi(k) \leq 1-\frac{1}{k}$.

- $\varphi(k)=1-\frac{1}{k}$ if and only if $\varphi^{\prime}(1)=1$,

- $\lim _{k \rightarrow \infty} \psi(k)=1$.

The minimal displacement problem for the class $\mathcal{L}$ is the task to find in the open form or to evaluate functions $\varphi$ and $\psi$ for concrete sets or spaces.

From our initial considerations we always have

$$
\psi_{X}(k) \leq \varphi_{X}(k) \leq 1-\frac{1}{k} .
$$

Let us conclude with the example showing that the above estimate is, in some spaces sharp.

Example 5. Let $X=c_{0}$ and let for $t \in[0, \infty], \alpha(t)=\min [t, 1]$. Define the mapping $T: B \rightarrow B$ as

$$
T x=T\left(x_{1}, x_{2}, x_{3}, \ldots\right)=\left(1, \alpha\left(k\left|x_{1}\right|\right), \alpha\left(k\left|x_{2}\right|\right), \alpha\left(k\left|x_{3}\right|\right), \ldots\right) .
$$

Thus, $T \in \mathcal{L}(k)$ and for any $x=\left(x_{1}, x_{2}, x_{3}, \ldots\right) \in B,\|x-T x\|>1-\frac{1}{k}$. Indeed, the reverse inequality implies $x_{1} \geq \frac{1}{k}$ and $\alpha\left(k\left|x_{1}\right|\right)=1$. Thus $x_{2} \geq \frac{1}{k}, \alpha\left(k\left|x_{2}\right|\right)=1$ and consequently for the same reason $x_{i} \geq \frac{1}{k}$ for all $i=1,2,3, \ldots$, which contradicts $x \in c_{0}$. It shows also that the minimal displacement of $T, d(T)=1-\frac{1}{k}$ is not achieved by $T$ at any point of $B$.

Further examples and informations about the properties of function $\varphi$ are going to be shown in the coming sections.

\subsection{Optimal retraction problem}

Suppose for have a space $X$ and the unit ball $B$. Suppose we have found a lipschitzian mapping $T: B \rightarrow B$ with $d(T)>0$. Using the trick presented in Section 2 we can construct a retraction $R: B \rightarrow S$. It is only a technicality to prove that the retraction obtained is, in this case, lipschitzian. Possibly, there are many other ways to construct such retractions.

The optimal retraction problem is the task to find or evaluate the constant characterizing given space $X$, traditionally denoted $k_{0}(X)$ and defined as

$$
k_{0}(X)=\inf [k: \text { There exists a retraction } R: B \rightarrow S \text { of class } \mathcal{L}(k)] .
$$

The problem was for the first time set again in ([11]). Since 1973 a number authors tried to get good estimates. The progress is very slow. Here, we conclude with, probably the simplest known, example. 
Example 6. Let $X=C[0,1]$. The mapping $A: B \rightarrow C[0,1]$ defined by

$$
(A f)(t)=|f(t)+1-2(1-\|f\|) t|-1+2(1-\|f\|) t
$$

is lipschitzian, $A \in \mathcal{L}(5)$ and for all $f \in S$, we have $A f=f$. We leave to the reader to check that for all $f \in B,\|f\|$ is separated from zero. More precisely one can prove that

$$
\inf [\|A f\|: f \in B]=\frac{1}{7} .
$$

Now, we can define the retraction $R: B \rightarrow S$ as

$$
R f=\frac{A f}{\|A f\|}=P(7 A f),
$$

where $P: C[0,1] \rightarrow B$ stands for radial projection. Since $P \in \mathcal{L}(2)$ and $A \in \mathcal{L}(5)$, we get $R \in \mathcal{L}(70)$.

In the terminology introduced above we can conclude with the observation that $k_{0}(C[0,1]) \leq 70$. Obviously, this estimate is not precise. More detailed discussion is presented in Section 8.

\subsection{Optimal homotopy problem}

Once we have a retraction $R: B \rightarrow S$, using the way indicated in Section 2 one can define the homotopy $H:[0,1] \times S \rightarrow S$ joining identity with a constant map, $H(t, x)=R((1-t) x)$. If $R \in \mathcal{L}(k)$ such homotopy is satisfies

$$
\|H(t, x)-H(s, y)\| \leq k(|t-s|+\|x-y\|) .
$$

The optimal homotopy problem deals with finding, for various spaces, homotopies $H:[0,1] \times S \rightarrow S$ satisfying

$$
\|H(t, x)-H(s, y)\| \leq A|t-s|+B\|x-y\| .
$$

with relatively small constants $A, B$.

The problem is discussed among specialists, but there are not many works dedicated to it. However, some tricks with such homotopies are used in connection with finding optimal retractions. Let us present here an example of such homotopy taken from $([13])$.

Example 7. Let $X=L^{1}(0,1)$. For any $c \in[0,1]$ and $f \in S$, define

$$
t_{f}(t)=\sup \left[t: \int_{0}^{t}|f| d t=c\right] \text {. }
$$

Set

$$
H(c, f)(t)=\left\{\begin{array}{cc}
|f(t)| & \text { if } t \leq t_{f}(c) \\
f(t) & \text { if } t>t_{f}(c) .
\end{array}\right.
$$

This homotopy joins each function $f \in S$ with its absolute value $|f|, H(0, f)=f$ and $H(1, f)=|f|$. The word "homotopy" is justified by the fact that

$$
\|H(c, x)-H(d, y)\| \leq 2|c-d|+2\|x-y\|,
$$


which we leave to prove to the reader. The homotopy $H$ keeps all the nonnegative functions invariant. If $f \in S^{+}=[f \in S: f \geq 0]$ then $H(c, f)=f$ for all $c \in[0,1]$. Since $S^{+}$is a convex subset of the sphere $S$, it is contractible to any of its point $g$ by the natural homotopy $G(c, f)=(1-c) f+c g$. Gluing up two homotopies $H$ and $G$ we get a homotopy joining identity on $S$ with a constant map. We leave to the reader showing that such homotopy is lipschitzian and finding constants $A$ and $B$.

\section{Solutions of qualitative questions}

Most of the problems, ideas and discussions presented in the Sections above were based on intuition coming from individual examples. The first general breakthrough came in 1979 after the paper of B. Nowak ([28]). The result stated that for certain class of Banach spaces (those admitting weakly continuous duality mapping) there are lipschitzian retractions of the ball onto sphere. In our terminology, if $X$ belongs to this class then $k_{0}(X)<\infty$.

The complete much stronger solution came 4 years later in ([5]),

Theorem 9 (Y. Benyamini and Y. Sternfeld). For any Banach space $X$, there exists a lipschitzian retraction $R: B \rightarrow S$.

In our terminology it means that for any Banach space $X, k_{0}(X)<\infty$. The title of the paper states the result in indirect form. Spheres in infinitely dimensional Banach spaces are Lipschitz contractible. Then the solution of retraction problem is obtained by standard tricks. The proof is very technical but universal. It works for all the Banach spaces regardless of regularity. Following this result, the "trivial theorem" and its consequences presented in Preliminaries, take much stronger form:

Theorem 10. For any Banach space $X$ of infinite dimension the following five statements are true and equivalent:

A) For any $k>1$ there exists a k-lipschitzian mapping $T: B \rightarrow B$ with $d(T)>0$,

B) $\psi_{X}(k)>0$ for all $k>1$,

C) The unit sphere $S$ is the lipschitzian retract of $B$,

D) $k_{0}(X)<\infty$,

E) $S$ is Lipschitz contractible.

It is not clearly mentioned in ([5]) but it was noticed by other authors working in the field that there is something more in the proof. The proof is constructive and close analysis of it allows to formulate much stronger

Conclusion 1. There exists an universal constant $k_{0}$ such that for any Banach space $X$ there exists a retraction $R: B \rightarrow S$ with $k(R) \leq k_{0}$. In other words, $\sup k_{0}(X)<$ $\infty$ where supremum is taken over the whole category of Banach spaces.

Final step concerning not only unit ball but all closed convex and bounded sets has been made by P.K. Lin and Y. Sternfeld ([25]): 
Theorem 11 (P.K. Lin and Y. Sternfeld). For any Banach space $X$, any closed, bounded, convex but noncompact set $C \subset X$ and any $k>1$ there exists a mapping $T: C \rightarrow C$ of class $\mathcal{L}(k)$ such that $d(T)>0$.

In our terminology it means that for such sets $C$ we have $\varphi_{C}(k)>0$ for all $k>1$.

The above results close, in some sense, the qualitative part of the theory. All the proofs are so technically complicated that do not allow to make estimations, neither for the size of $k_{0}$ (or $k_{0}(X)$ for particular space $X$ ) nor for $\psi(k)$ (or $\varphi_{C}(k)$ for particular set $C$ ). Good estimates require individual approach. In what follows we will present some constructions.

\section{Results concerning minimal displacement}

Once we know that $\varphi_{C}(k)>0$ for $k>1$ and all noncompact convex sets $C$, the main question in the field is to evaluate this function. For simplicity we shall concentrate mostly on the case of unit ball $B$ and the function $\psi_{X}$. As it was shown above we have

and (see Example 5)

$$
\psi_{X}(k) \leq 1-\frac{1}{k}
$$

$$
\psi_{c_{0}}(k)=1-\frac{1}{k}
$$

In general we call the set $C$ extremal (with respect to minimal displacement) if

$$
\varphi_{C}(k)=\left(1-\frac{1}{k}\right) r(C) .
$$

In this terminology the unit ball in $c_{0}$ is extremal. There are many other spaces having extremal balls. Among them are spaces of continuous functions $C[a, b]$, continuously differentiable functions $C^{n}[a, b]$ with standard and modified uniform norms. Also it is known (see [7], unpublished) that all the subspaces of $C[a, b]$ of finite codimension are of extremal balls. Here are three examples of extremal sets and balls.

Example 8. Let $X=C[0,1]$ and let $K \subset C[0,1]$ be the closed convex set defined by

$$
K=[x: 0=x(0) \leq x(t) \leq x(1)=1] .
$$

We have $r(K)=\operatorname{diam} K=1$. Let $e \in K$ be the identity function, $e(t)=t$ for $t \in[0,1]$ and let $\alpha \in K$ be chosen arbitrary with $\alpha \neq e$. Define the mapping $T_{\alpha}: K \rightarrow K$ by

$$
T_{\alpha} x(t)=(\alpha \circ x)(t)=\alpha(x(t)) .
$$

The regularity of $T_{\alpha}$ is determined by $\alpha$. If $\alpha$ is lipschitzian with constant $k$, $|\alpha(t)-\alpha(s)| \leq k|t-s|$ then $T_{\alpha} \in \mathcal{L}(k)$. Now, we observe that since each $x \in K$ 
takes all the values between 0 and 1 , we have

$$
\begin{aligned}
\left\|x-T_{\alpha} x\right\| & =\max [|x(t)-\alpha(x(t))|: t \in[0,1]] \\
& =\max [|s-\alpha(s)|: s \in[0,1]]=\|e-\alpha\|=\text { const }>0 .
\end{aligned}
$$

Thus, all mappings $T_{\alpha}$ have constant positive displacement $d\left(T_{\alpha}\right)=d_{\alpha}=\|e-\alpha\|$. If, for any $k>1$ we select the function $\alpha_{k}(t)=\min [k t, 1]$ and define $T_{k}=T_{\alpha_{k}}$, we get, $T_{k} \in \mathcal{L}(k)$ and for all $x \in K$,

$$
\left\|x-T_{k} x\right\|=\left\|e-\alpha_{k}\right\|=1-\frac{1}{k}
$$

This means that $K$ is extremal. It is also worth to observe that the family $T_{k}, k>$ 1 of mappings realizing the extremal minimal displacement form the semigroup, $T_{k} \circ T_{l}=T_{k l}$.

There are many constructions showing that in general $\psi_{C[a, b]}=1-\frac{1}{k}$. We leave to the reader justification of the next example.

Example 9. Let

$$
\beta(t)=\left\{\begin{array}{cc}
-1 & \text { for } t \leq-1 \\
t & \text { for }-1 \leq t \leq 1 \\
1 & \text { for } t \geq 1
\end{array}\right.
$$

and let $y:[a, b] \rightarrow(-\infty,+\infty)$ be an arbitrary function such that $y(a) \leq-2$ and $y(b) \geq 2$. Then, for $k>1$, the mapping $T: B \rightarrow B$ defined as

$$
T x(t)=\beta(k(x(t)+y(t)))
$$

is of class $\mathcal{L}(k)$ with $d(T)=1-\frac{1}{k}$.

One more example of an extremal set is in $L^{1}(0,1)$.

Example 10. Let $S^{+}$be the positive part of the unit sphere,

$$
S^{+}=\left[f: f \geq 0, \int_{0}^{1} f(t) d t=1\right] .
$$

Clearly $S^{+}$is closed convex with $r\left(S^{+}\right)=2$. For any $k>1$ and $f \in S^{+}$define the point $t_{f} \in(0,1)$,

$$
t_{f}=\inf \left[t: \int_{0}^{t} f(s) d s=1-\frac{1}{k}\right] .
$$

It is a technicality to show that the mapping

$$
T f(t)=\left\{\begin{array}{cc}
0 & \text { for } 0 \leq t<t_{f} \\
k f(t) & \text { for } t_{f} \leq t \leq 1
\end{array}\right.
$$

is $k$-lipschitzian and that for all $f \in S^{+}$

$$
\|f-T f\|=d(T)=2\left(1-\frac{1}{k}\right) .
$$

Thus $S^{+}$is extremal. 
There are many other examples of extremal sets and balls. The nonextremal case is more complicated. It is known that some spaces have balls which are not extremal. For example such are all uniformly convex spaces. However, so far, there is not known a space $X$ which is not extremal and for which the exact formula for $\psi_{X}$ is determined in the open form. Two spaces are of special interest; the Hilbert space $H$ and $l^{1}$. The Hilbert space, being geometrically the most regular should have relatively small, if not the smallest function $\psi_{H}$.

The following evaluation can be found in ([11]),

$$
\psi_{H}(k) \leq\left(1-\frac{1}{k}\right) \sqrt{\frac{k}{k+1}}<1-\frac{1}{k} .
$$

For over 35 years it was not shown if this estimate is sharp and no one better was found. In our opinion answering this question is one of the most important challenge in the field.

The case of $l^{1}$ is special. In many aspects of the geometry of Banach spaces $l^{1}$ is considered to be irregular and have "very square" balls. It can be shown (see [10],[13]) that, as in $L^{1}$, (see Example 10) the positive part $S^{+}$in $l^{1}$ is extremal. However the whole unit ball $B$ is not. The following estimate holds,

$$
\psi_{l^{1}}(k) \leq\left\{\begin{array}{cc}
\frac{2+\sqrt{3}}{4}\left(1-\frac{1}{k}\right) & \text { for } 1 \leq k \leq 3+2 \sqrt{3} \\
\frac{k+1}{k+3} & \text { for } k>3+2 \sqrt{3}
\end{array}\right.
$$

Consequently $\psi_{H}^{\prime}(1) \leq \frac{1}{\sqrt{2}}$ and $\psi_{l^{1}}^{\prime}(1) \leq \frac{2+\sqrt{3}}{2}$.

The above estimates are related to the early questions which has been raised in [11]. Does there exist a space $Z$ such that $\psi_{Z} \leq \psi_{X}$ for all spaces $X$ ? Does there exist a space $Z$ such that $\psi_{Z}^{\prime}(1) \leq \psi_{X}^{\prime}(1)$ for all spaces $X$ ? Again, for over 35 years the progress in answering such questions is almost nil.

Some special subclasses of $\mathcal{L}(k)$ were also considered in relation to the optimal retraction problems. Let us mention two which will be used in the next section. For details see [10].

First, let us restrict ourselves to the class of mappings $T$ transforming the unit ball into its boundary, the unit sphere $S, T: B \rightarrow S$. Define the characteristic of minimal displacement for this class as:

$$
\psi_{B \rightarrow S}(k)=\sup [d(T): T: B \rightarrow S, T \in \mathcal{L}(k)] .
$$

In some spaces like $c_{0}, C[a, b]$ we have $\psi_{B \rightarrow S}(k)=\psi(k)=1-\frac{1}{k}$ (see examples above). However it is not so in more regular spaces. For example for Hilbert space $H$, we have

$$
\psi_{B \rightarrow S}(k) \leq\left(1-\frac{1}{k}\right)^{\frac{3}{2}}
$$

with $\psi_{B \rightarrow S}^{\prime}(1)=0<\psi^{\prime}(1)$.

Second, consider the class of all transformations $T: B \rightarrow X$ sending all the boundary points to the origin, $T(S)=\{0\}$. Again the characteristic of minimal 
displacement can be defined as

$$
\left.\psi_{S \rightarrow 0}(k)=\sup [d(T): T: B \rightarrow X, T(S)=\{0\}, T \in \mathcal{L}(k))\right] .
$$

For such characteristic we have

$$
\psi_{S \rightarrow 0}(k) \leq \min \left[1, \frac{k}{2} \psi(k)\right] \leq \min \left[1, \frac{k-1}{2}\right],
$$

with $\psi_{S \rightarrow 0}^{\prime}(1) \leq \frac{1}{2} \psi^{\prime}(1)$ and $\psi(k)=1$ for sufficiently large $k$. An imprecise estimate for the last claim is $k>k_{0}+1$, where $k_{0}$ is the universal constant mentioned in the conclusion to the Benyamini-Sternfeld Theorem. Indeed, if $R: B \rightarrow S$ is a $k$-lipschitzian retraction, then $T: B \rightarrow X$ defined as $T x=x-R x$, satisfies $T(S)=\{0\}, T \in \mathcal{L}(k+1)$ and $d(T)=1$.

\section{Results on optimal retraction problem}

The investigations concerning the optimal retraction problems concentrate in general on finding nice estimates of $k_{0}(X)$ for particular spaces $X$. It is hard to say that there are any general methods to obtain such evaluations. Most of the work is done by applying special tricks to construct examples. There is no space $X$ for which exact value of $k_{0}(X)$ is known. Here we present some basic facts and list the most interesting estimates.

First observe that the constant $k_{0}(X)$ can not be too small.

Claim 1. For any Banach space $X, k_{0}(X) \geq 3$.

To observe this, consider a lipschitzian retraction $R: B \rightarrow S, R \in \mathcal{L}(k)$.Define $T=-R$ observe that $T^{2}=R$ and take any $x \in B$. Let $d(x)=\|x-T x\|=$ $\|x+R x\|$. Now we have

$$
2=\left\|T^{2} x-T x\right\| \leq k\|T x-x\|=k d(x) .
$$

Since for any $\varepsilon>0, x \in B$ can be selected so that $d(x) \leq \psi(k)+\varepsilon$, we get

$$
2 \leq k \psi(k) \leq k\left(1-\frac{1}{k}\right)=k-1
$$

which implies our claim. It is not clear whether the above estimate is sharp. Certainly for some regular spaces we can get better bound. Observe that our construction the mapping $T$ maps the segment $[x, T x]$ onto a lipschitzian (just rectifiable) curve which joins two antipodal points, $T x$ and $T^{2} x=R x=-T x$. The minimal length of such curve is called the girth of the sphere, is denoted by $g(X)$ and satisfies $g(X) \geq 2$. For some spaces $g(X)=2$ but for some (e.g. uniformly convex) we have $g(X)>2$. For example, for the Hilbert space we have $g(X)=\pi$. For details on the girth see $([31])$. We leave to the reader to observe that a technical refinement of the arguments we used above lead to the sharper inequality

$$
g(X) \leq k \psi_{B \rightarrow S}(k) .
$$


Using the estimate from the previous section, for the Hilbert space we get

$$
\pi \leq k\left(1-\frac{1}{k}\right)^{\frac{3}{2}}
$$

which implies $k_{0}(H) \geq 4.5 \ldots$.

The evaluation $k_{0}(X) \geq 3$ seems to be imprecise even for spaces with $g(X)=2$. For example we have $g\left(l^{1}\right)=2$, but $k_{0}\left(l^{1}\right) \geq 4$ ( see [7]).

There are more results concerning estimates from above. There are not satisfactory evaluations for the maximal $k_{0}$. All the attempts to evaluate it ends at the level of high thousands. Much better situation is observed for particular spaces or some classes of spaces. For years, step by step improvements has been done by a number of authors. The first estimates placed $k_{0}(X)$ for classical Banach spaces between 10 and 40. The first space for which it was known that its retraction constant does not exceed 10 was $L^{1}(0,1)$. For a long time the best estimate was $k_{0}\left(L^{1}(0,1)\right) \leq 9.43$, where the last number was a solution of certain equation. The general situation till 2002 is discussed in [10] and [19] (Chapter 17).

All the results of this type are obtained by producing concrete examples of mappings. Let us present here some recent constructions of retractions with relatively small Lipschitz constants. The examples are selected on the bases of their simplicity. The best known estimates obtained via longer constructions will be only mentioned.

Let us begin with spaces $l^{1}$ and $L^{1}(0,1)$. An original construction presented in [1] and [2] shows that

$$
4 \leq k_{0}\left(l^{1}\right) \leq 8
$$

On the basis of slightly modified technique the same estimate from above has been established also for $L^{1}(0,1)$ and some other spaces having similar geometrical properties (see . [14]). Here is the $L^{1}$ version.

Example 11. Consider the unit ball $B$ and the unit sphere $S$ in $L^{1}(0,1)$. For technical reason let us assume that all the functions $h \in L^{1}(0,1)$ are extended to the negative axis by putting $h(t)=0$ for $t<0$. For any function $h \in B$ define the number

$$
t_{h}=\inf \left[t: \int_{t}^{1}|h(s)| d s \leq 1-\|h\|\right] \text {. }
$$

Observe that

and that

$$
t_{h}=0 \text { if }\|h\| \leq \frac{1}{2}
$$

$$
\int_{t_{h}}^{1}|h(s)| d s=1-\|h\| \text { if } \frac{1}{2} \leq\|h\| \leq 1 .
$$

Next define the mapping $Q: B \rightarrow \frac{1}{2} B$ by

$$
Q h(t)=\left\{\begin{array}{cc}
0 & \text { if } t<t_{h}, \\
h(t) & \text { if } t_{h} \leq t .
\end{array} .\right.
$$

and notice that:

$$
Q h=h \text { and consequently }\|Q h\|=\|h\|, \text { if }\|h\| \leq \frac{1}{2},
$$




$$
\begin{gathered}
\|Q h\|=1-\|h\|, \text { if }\|h\| \geq \frac{1}{2} \text { and consequently } Q(S)=\{0\}, \\
(I-Q) h=0 \text { and consequently }\|(I-Q) h\|=0 \text {, if }\|h\| \leq \frac{1}{2}, \\
\|(I-Q) h\|=\|h\|-\|Q h\|=2\|h\|-1, \text { if }\|h\| \geq \frac{1}{2} .
\end{gathered}
$$

The last observation comes from the fact that functions $Q h$ and $(I-Q) h$ have disjoint supports. Now let us evaluate the Lipschitz constants of $Q$ and $I-Q$. Restrictions of these mappings to the ball $\frac{1}{2} B$ have Lipschitz constants 1 and 0 respectively. Take any two functions $f, g \in B$ with norms $\|f\| \geq \frac{1}{2},\|g\| \geq \frac{1}{2}$ and just for technical reason assume that $t_{f} \leq t_{g}$. We have

$$
\begin{aligned}
\|(I-Q) f-(I-Q) g\|= & \int_{0}^{t_{f}}|f(s)-g(s)| d s+\int_{t_{f}}^{t_{g}}|g(s)| d s \\
= & \int_{0}^{t_{f}}|f(s)-g(s)| d s+\int_{t_{f}}^{1}|g(s)| d s-\int_{t_{g}}^{1}|g(s)| d s \\
= & \int_{0}^{t_{f}}|f(s)-g(s)| d s+\int_{t_{f}}^{1}|g(s)| d s-1+\|g\| \\
= & \int_{0}^{t_{f}}|f(s)-g(s)| d s+\int_{t_{f}}^{1}|g(s)| d s \\
& -\int_{t_{f}}^{1}|f(s)| d s-\|f\|+\|g\| \\
\leq & \int_{0}^{1}|f(s)-g(s)| d s+|\|g\|-\|f\|| \\
\leq & 2\|f-g\| .
\end{aligned}
$$

Consequently

$$
\begin{aligned}
\|Q f-Q g\| & =\|(f-g)-((I-Q) f-(I-Q) g)\| \\
& \leq\|f-g\|+\|(I-Q) f-(I-Q) g\| \\
& \leq 3\|f-g\| .
\end{aligned}
$$

So $Q$ is 3-lipschitzian and $I-Q$ is 2-lipschitzian. Now let $A: L^{1}(0,1) \rightarrow L^{1}(0,1)$ be the isometry defined by

$$
A h(t)=2 h(2 t-1) .
$$

Observe that if $\|h\| \leq \frac{1}{2}$ then the support of $A h$ is contained in the interval $\left[\frac{1}{2}, 1\right]$. If $\|h\| \geq \frac{1}{2}$, then support of $A Q h$ is contained in $\left[\frac{t_{h}+1}{2}, 1\right]$ and since the support of $(I-Q) h$ is contained in $\left[0, t_{h}\right]$, the functions $A Q h$ and $(I-Q) h$ have disjoint supports. Knowing the above we can construct the retraction $R: B \rightarrow S$ by

$$
R h=\left\{\begin{array}{cl}
2(1-2\|h\|) \chi_{\left[0, \frac{1}{2}\right]}+2 A h & \text { if }\|h\| \leq \frac{1}{2} \\
(I-Q) h+2 A Q h & \text { if }\|h\| \geq \frac{1}{2} .
\end{array}\right.
$$


Where $\chi_{\left[0, \frac{1}{2}\right]}$ denotes the characteristic function of $\left[0, \frac{1}{2}\right]$. The both formulas coincide if $\|h\|=\frac{1}{2}$. If $\|h\| \leq \frac{1}{2}$, then

$$
\begin{aligned}
\|R h\| & =\int_{0}^{\frac{1}{2}} 2(1-2\|h\|) d s+4 \int_{\frac{1}{2}}^{1}|h(2 s-1)| d s \\
& =1-2\|h\|+2 \int_{0}^{1}|h(s)| d s=1
\end{aligned}
$$

and if $\|h\| \geq \frac{1}{2}$, then also

$$
\begin{aligned}
\|R h\| & =\|(I-Q) h\|+2\|A Q h\| \\
& =2\|h\|-1+2(1-\|h\|)=1 .
\end{aligned}
$$

Thus indeed $R: B \rightarrow S$ with $R=I$ on $S$. Moreover, for $f, g$ with $\|f\| \leq \frac{1}{2},\|g\| \leq \frac{1}{2}$ we have

$$
\|R f-R g\|=2|\|f\|-\|g\||+2\|A f-A g\| \leq 4\|f-g\|,
$$

while if $\|f\| \geq \frac{1}{2},\|g\| \geq \frac{1}{2}$,

$$
\begin{aligned}
\|R f-R g\| & =\|(I-Q) f-(I-Q) g\|+2\|A Q f-A Q g\| \\
& \leq 2\|f-g\|+6\|f-g\|=8\|f-g\|
\end{aligned}
$$

If $\|f\| \geq \frac{1}{2}$ and $\|g\| \leq \frac{1}{2}$ then there exists a number $\alpha \in[0,1]$ such that $\|(1-\alpha) f+\alpha g\|=\frac{1}{2}$. Then we have

$$
\begin{aligned}
\|R f-R g\| & \leq\|R f-R((1-\alpha) f+\alpha g)\|+\|R((1-\alpha) f+\alpha g)-R g\| \\
& \leq 8 \alpha\|f-g\|+4(1-\alpha)\|f-g\| \leq 8\|f-g\| .
\end{aligned}
$$

Thus the inequality

$$
\|R f-R g\| \leq 8\|f-g\|
$$

holds for all $f, g \in B$ meaning $R$ is 8-lipschitzian.

The above implies that

$$
3 \leq k_{0}\left(L^{1}(0,1)\right) \leq 8
$$

The left inequality is weaker than this for $l^{1}$ and has not been improved yet.

Let us pass to the space of continuous functions $C[0,1]$. The old estimate (see e.g. [10])

$$
3 \leq k_{0}(C[0,1]) \leq 4(1+\sqrt{2})^{2}=23.31 \ldots
$$

has been recently improved in [29] to

$$
3 \leq k_{0}(C[0,1]) \leq 4(2+\sqrt{3})=14.92 \ldots
$$

and it is the best known estimate for this space. Better estimates has been obtained for the subspace $C_{0}[0,1] \subset C[0,1]$ consisting of all functions vanishing at zero, $f(0)=0$. Here is an elementary construction. 
Example 12. Let $X=C_{0}[0,1]$ be the space of continuous functions on $f:[0,1] \rightarrow R$ vanishing at zero, $f(t)=0$ with standard uniform norm $\|f\|=\max |f(t)|$. Consider the function $\Lambda:[0, \infty] \rightarrow\left[0, \frac{3}{2}\right]$ defined by

$$
\Lambda(t)=\left\{\begin{array}{cc}
3 t & \text { for } 0 \leq t \leq \frac{1}{2} \\
3(1-t) & \text { for } \frac{1}{2} \leq t \leq 1 \\
0 & \text { for } t \geq 1
\end{array} .\right.
$$

Let $T_{0}: C_{0}[0,1] \rightarrow \frac{3}{2} B \subset C_{0}[0,1]$ be defined by

$$
\left(T_{0} f\right)(t)=\Lambda(|f(t)|+t) .
$$

Observe that $T_{0}$ satisfies Lipschitz condition with constant $k=3$. Also observe that for any function $f \in C_{0}[0,1]$ there exists a point $t_{1} \in(0,1)$ such that $\left|f\left(t_{1}\right)\right|+t_{1}=\frac{1}{2}$. Hence, for all $f \in C_{0}[0,1]$ we have

$$
\left\|T_{0} f-f\right\| \geq\left|\left(T_{0} f\right)\left(t_{1}\right)-f\left(t_{1}\right)\right| \geq\left|\left(T_{0} f\right)\left(t_{1}\right)\right|-\left|f\left(t_{1}\right)\right|=\frac{3}{2}-\frac{1}{2}+t_{1}>1 .
$$

For functions satisfying with $\|f\| \geq 1$, we have another observation. If there is a point $t_{2}$ such that $f\left(t_{2}\right)<-1$, we have $\left(T_{0} f\right)\left(t_{2}\right) \geq 0$ and for any point $t_{3}$ such that $f\left(t_{3}\right)>1$ we have $\left(T_{0} f\right)\left(t_{3}\right)=0$. Obviously, at least one of the points $t_{2}$ or $t_{3}$ satisfying the above does exist. In both cases we also have $\left\|T_{0} f-f\right\| \geq 1$.

Now, define the mapping $T_{1}: \frac{3}{2} B \rightarrow \frac{3}{2} B$,

$$
\left(T_{1} f\right)(t)=\left\{\begin{array}{cc}
\left(T_{0} f\right)(t) & \text { if }\|f\| \leq 1 \\
\min \left\{\left(T_{0} f\right)(t), 3\left(\frac{3}{2}-\|f\|\right)\right\} & \text { if } 1 \leq\|f\| \leq \frac{3}{2}
\end{array} .\right.
$$

The mapping $T_{1}$ is lipschitzian with Lipschitz constant $k=3$. For all $f \in \frac{3}{2} B$, $\left\|T_{1} f-f\right\| \geq 1$. Also $T_{1}$ sends the sphere $\frac{3}{2} S$ into the origin. In other words $T_{1}\left(\frac{3}{2} S\right)=$ $\{0\}$. Consequently the mapping $T: B \rightarrow B$ defined by

$$
T(f)=\frac{2}{3} T_{1}\left(\frac{3}{2} f\right),
$$

has the same Lipschitz constant $k=3$, satisfies $\left\|T_{1} f-f\right\| \geq \frac{2}{3}$ for all $f \in B$ and sends the unit sphere $S$ to the origin, $T(S)=\{0\}$.

Now we can define the retraction $R: B \rightarrow S$. Put

$$
R f=\frac{f-T f}{\|f-T f\|}=P\left(\frac{3}{2}(f-T f)\right) .
$$

Here $P: C_{0}[0,1] \rightarrow B$ denotes the radial projection,

$$
P f=\left\{\begin{array}{cl}
f & \text { if }\|f\| \leq 1 \\
\frac{f}{\|f\|} & \text { if }\|f\|>1
\end{array} .\right.
$$

It is known that the Lipschitz constant of $P$ equals 2,

$$
\|P f-P g\| \leq 2\|f-g\| .
$$


Thus we have

$$
\begin{aligned}
\|R f-R g\| & =\left\|P\left(\frac{3}{2}(f-T f)\right)-P\left(\frac{3}{2}(g-T g)\right)\right\| \\
& \leq 3\|(f-T f)-(g-T g)\| \\
& =3\|f-g\|+3\|T f-T g\| \\
& \leq 3\|f-g\|+9\|f-g\|=12\|f-g\| .
\end{aligned}
$$

Thus we conclude with $k_{0}\left(C_{0}[0,1]\right) \leq 12$.

The above estimate is not optimal. More technical approach has been presented in [15] with better conclusion $k_{0}\left(C_{0}[0,1]\right) \leq 7$. The strongest known and the most general result in this context is

$$
3 \leq k_{0}\left(C B_{z}(M)\right) \leq 2(2+\sqrt{2})=6.83 \ldots
$$

where $M$ is an arbitrary connected metric space consisting of more then one point, $z \in M$ and $C B_{z}(M)$ is the space of bounded continuous functions vanishing at $z$ (see [30]).

The case of Hilbert space is very interesting. The geometry of Hilbert space $H$ is very regular and, probably, this causes difficulties in finding explicit examples of retractions $R: B \rightarrow S$. Moreover, as mentioned above an exact formula for the characteristic of minimal displacement $\psi_{H}(k)$ is unknown does not help in finding good estimates.

The first estimate $k_{0}(H) \leq 64.25$ has been presented in [22]. Then, step by step, there were several improvements. Let us mention a constructive example from [8], $k_{0}(H)<32.26$. and

$$
k_{0}(H) \leq 28.99
$$

presented in [4]. The above estimate seems to be very rough but none better is known. There are some findings which suggest so. These are some inequalities which tie unknowns, $k_{0}(H)$ and some values of $\psi_{H}(k)$. Here are two samples (see [10])

$$
\begin{aligned}
& k_{0}(H) \psi_{H}^{\prime}(1) \leq 8, \\
& k_{0}(H) \psi_{H}(3) \leq \frac{16}{3} .
\end{aligned}
$$

Finding the best estimates for $k_{0}(H)$ and $\psi_{H}^{\prime}(1)$ is in our opinion the main and difficult challenge in the field.

Finally, we should mention some results related to more general case. As mentioned above a good and reasonable estimate for $k_{0}=\sup k_{0}(X)$ with respect to all Banach spaces $X$ is practically unknown. There are several estimates, some similar to the last ones presented above for Hilbert space which are in the "folklore" of the subject. Some can be found in ([10]). For example, for any Banach space $X$ we have

$$
\begin{array}{r}
k_{0}(X) \psi_{X}^{\prime}(1) \leq 16(1+\sqrt{2})^{2}=93.25 \ldots, \\
k_{0}(X) \psi_{X}(1+\sqrt{2}) \leq 16(2+\sqrt{2})=54.62 \ldots .
\end{array}
$$


Also, there are some more general inequalities which evaluate $k_{0}(X)$, as

$$
k_{0}(X) \leq 3 k(P) \min \left[\frac{k+2}{2 \psi(k)-1}: k>\psi^{-1}\left(\frac{1}{2}\right)\right] \text {. }
$$

Here $k(P) \leq 2$ is the Lipschitz constant for the radial retraction of $X$ onto the unit ball $B$. Applying this estimate for extremal spaces having $\psi(k)=1-\frac{1}{k}$ we get

$$
k_{0}(X) \leq 3 k(P)(2+\sqrt{2})^{2}=k(P) 34.97 \ldots<70 .
$$

The above is not the best known estimate in the class of extremal spaces. There were several improvements which so far ended with (see [4])

$$
\psi_{X}(k)=1-\frac{1}{k} \Longrightarrow k_{0}(X) \leq 30.84 \ldots .
$$

\section{Final remarks}

The presented direction of investigation is, as we were trying to show, full of open problems. Even questions raised in the first paper [11] almost forty years ago are still open. The progress is slow. Most of the results come from some tricky constructions invented by a number of authors. There are not to many general theorems and rules to proceed. Everything depends on individual creativity.

There are also directions of research related to the problem which we did not mention. To end our presentation we would like to turn the readers attention to one, which gain some popularity among researchers.

All the questions raised for lipschitzian mappings can be translated to some other classes. Let us recall that the continuous mapping $T: C \rightarrow C$ is said to be $\alpha$-set contraction if for every subset $D \subset C, \alpha(T(D)) \leq k \alpha(D)$ where $\alpha$ is the Kuratowski's measure of noncompactness $\alpha(D)=\inf d$ that $D$ can be divided into finite number of sets of diameter smaller then $d$. All the $k$-lipschitzian mappings are $k$-set contractions. However this class is wider because it is closed with respect some compact perturbations. Surprisingly optimal retraction problem for this class of mappings and for some spaces is solved completely.

There are spaces (e.g. $C[0,1])$ such that for any $\varepsilon>0$ there exists a retraction $R: B \rightarrow S$ being $(1+\varepsilon)$-set contraction [32]. There are even spaces for which there are retractions of the ball onto sphere constructed as a compact perturbations of a nonexpansive map. More informations about this direction can be found in an expository article [3].

\section{References}

[1] M. Annoni, Retrazioni e minimo spostamento negli spazi di Banach, Degree Thesis, (2005).

[2] M. Annoni, E. Casini, An upper bound for the Lipschitz retraction constant in $l_{1}$, Studia Math. 180 (2007), 73-76. 
[3] J. Appell, N. Erzakova, S. Falcon Santana, M. Väth, On some Banach space constants arising in nonlinear fixed point and eigenvalue theory, Fixed Point Theory Appl. (2004), no. $4,317-336$.

[4] M. Baronti, E. Casini, C. Franchetti, The retraction constant in some Banach spaces, J. Approx. Theory 120 (2003), no. 2, 296-308.

[5] Y, Benyamini, Y. Sternfeld, Spheres in infinite dimensional normed spaces are Lipschitz contractible, Proc. Amer. Math. Soc. 88 (1983), 439-445.

[6] P. Bohl, Über die Bewengung eines mechanishen Systems in der Nähe einer Gleichgewichtslage, J. Reine Angew. Math. 127 (1904), 179-276.

[7] K. Bolibok, Minimal displacement and retraction problems for balls in Banach spaces, Mariae Curie-Sklodowska University, Thesis (1999)

[8] K. Bolibok, Minimal displacement and retraction problems in infinite-dimensional Hilbert spaces, Proc. Amer. Math. Soc. 132 (2004), no. 4, 1103-111.

[9] L. E .J.Brouwer, Über Abbildungen von Mannigfaltigkeiten, Math. Ann. 71 (1912), 97115.

[10] K. Goebel, Concise course on fixed point theorems, Yokohama Publishers, (2002).

[11] K. Goebel, On the minimal displacement of points under lipschitzian mappings, Pacific J. Math. 48 (1973), 151-163

[12] K. Goebel, W. Kaczor Remarks on failure of Schauder's theorem in noncompact settings, Ann. Univ. Mariae Curie Skłodowska Sect. A 51 (1997), 99-108.

[13] K. Goebel, W. A. Kirk, Topics in metric fixed point theory, Cambridge University Press, (1990).

[14] K. Goebel, G. Marino, L. Muglia, R. Volpe, The retraction constant and minimal displacement characteristic of some Banach spaces, Nonlinear Anal. 67 (2007), 735-744.

[15] K.Goebel, Ł. Piasecki, A new estimate for the optimal retraction constant, Banach and Function Spaces II, Yokohama Publishers (2008), 77-83.

[16] K. Goebel, J. Wośko, Making a hole in the space, Proc. Amer. Math. Soc. 114 (1992), No.2, 475-476.

[17] S. Kakutani, Topological properties of the unit sphere of a Hilbert space, Proc. Imp. Acad. Tokyo 19 (1943), 269-271

[18] Y. Kannai, An elementary proof of no retraction theorem, Amer. Math. Monthly 88 (1981), 264-268.

[19] W. A. Kirk, B. Sims, Hanbook on metric fixed point theory, Kluwer Academic Publishers, (2001).

[20] V. Klee, Convex bodies and periodic homeomorphisms in Hilbert spaces, Trans. Amer. Math. Soc. 74 (1953), 10-43.

[21] V. Klee, Some topological properties of convex sets, Trans. Amer. Math. Soc. 78 (1955), $30-45$.

[22] T. Komorowski, J. Wośko, A remark on retracting of a ball ontoa sphere in an infinite dimensional Hilbert spase, Math. Scan. 67 (1990), 223-226.

[23] B. Knaster, K. Kuratowski, S. Mazurkiewicz, Ein Beweis des Fixpunktsatz für ndimensionale Simplexe, Fund. Math. 14 (1929), 132-137. 
[24] P. D. Lax, Change of variables in multiple integrals, Amer. Math. Monthly 106 (1999), 497-501.

[25] P. K. Lin, Y. Sternfeld, Convex sets with the Lipschitz fixed point property are compact, Proc. Amer. Math. Soc. 93 (1985), 633-639.

[26] D. Mauldin, The Scottish Book: mathematical problems from the Scottish Cafe, Birkhäuser, Boston, (1981).

[27] J. Milnor, Analytic proof of "Hairy Ball Theorem" and Brouwer Fixed Point Theorem, Amer. Math. Monthly, 85 (1978), 521-524.

[28] B. Nowak, On the Lipschitz retraction of the unit ball in infinite dimensional Banach space onto boundary, Bull. Acad. Polon. Sci. 27 (1979), 861-864.

[29] Ł. Piasecki, Retracting ball onto sphere in some Banach spaces, (preprint).

[30] Ł. Piasecki, Retracting ball onto sphere in $B C_{0}(\mathbb{R})$, Topol. Methods Nonlinear Anal. 33 (2009), No. 2, 307-314.

[31] J.J. Schaffer, Geometry of Spheres in Normed Spaces, Marcel Dekker (1976).

[32] J. Wośko, An example related to the retraction problem, Ann. Univ. Mariae CurieSkłodowska, Sect. A 45 (1991), 127-130.

Emanuele Casini

Dipartimento di Scienze Fisiche e Matematiche

Università degli Studi dell'Insubria

Via Vallegio 11

22100 Como

Italy

e-mail: emanuele.casini@uninsubria.it

Kazimierz Goebel

Instytut Matematyki, UMCS

20-031 Lublin

Poland

e-mail: goebel@hektor.umcs.lublin.pl

Lecture held by K. Goebel in the Seminario Matematico e Fisico on November 16, 2007.

Received: December 12, 2009.

Open Access This article is distributed under the terms of the Creative Commons Attribution Noncommercial License which permits any noncommercial use, distribution, and reproduction in any medium, provided the original author(s) and source are credited. 